\title{
Enunciación
}

\section{Creencias de futuros docentes sobre la gramática en la enseñanza de idiomas}

\author{
Future teachers' beliefs on grammar in language teaching

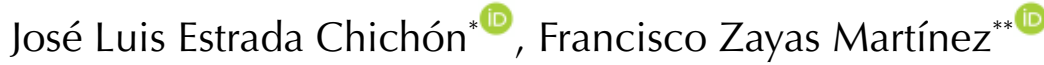

\begin{abstract}
Resumen
En este artículo se examinan las creencias de alumnos del grado en Educación Infantil en una universidad pública española de tamaño medio (20 000 estudiantes, aprox.) respecto del concepto de gramática en la enseñanza de idiomas. El trabajo se articula a partir de sus experiencias preuniversitarias, confrontándolas con su formación idiomática actual en la asignatura "Didáctica de la lengua extranjera en Educación Infantil: inglés". La metodología de investigación implica un enfoque mixto por combinar datos cuantitativos y cualitativos, a saber: i) utilizamos un cuestionario (Cook y Singleton, 2014) con el objeto de recopilar las opiniones de los estudiantes sobre el papel de la gramática en la enseñanza de idiomas; ii) organizamos un grupo focal para aclarar determinadas opiniones que son difícilmente entendibles solamente por medio de información cuantitativa. Asimismo, presentamos los principios fundamentales de su formación idiomática actual basada en mecanismos cognitivos de asimilación inconsciente del inglés según la enseñanza adquisitiva (Haidl, 1993, 1995) de leguas extranjeras, la cual evita la presentación explícita de reglas y normas gramaticales. Los resultados muestran opiniones similares entre los informantes, sobre todo para cuestionamientos relacionados con una formación que solo contemplaba el estudio formal de la gramática de la lengua meta. Asumen que sus pensamientos están fundamentados en una forma única de entender la enseñanza de idiomas que, además, coincide con la experimentada en primera persona durante etapas preuniversitarias: la instrucción formal. Con todo, a partir de la investigación-acción, parecen también acoger con satisfacción las posibilidades que brinda la enseñanza adquisitiva.
\end{abstract}

Palabras clave: gramática, enseñanza de idiomas, educación infantil.

\begin{abstract}
In this article, the beliefs of students pursuing a degree in primary education teaching at a medium-sized public Spanish university (approximately 20,000 students) are analysed in relation to the concept of 'grammar' in language teaching. This work is constructed on the basis of students' pre-university experiences in comparison to the students' current language training on the subject 'Didactics on foreign language in primary school: English'. The research methodology involves a mixed approach because it combines qualitative and quantitative data, specifically. First, we utilised a survey (Cook and Singleton, 2014) that aimed at gathering students' opinions on the role of grammar in language teaching. Second, we organised a focus group to clarify specific opinions which are poorly understood with only quantitative information. Furthermore, we present fundamental principles on current language training on the basis of the cognitive mechanisms of the unconscious assimilation of English according to foreign language acquisition teaching (Haidl, 1993, 1995), which avoids teaching grammatical rules and guidelines explicitly. The results exhibit similar opinions among respondents, especially in questions related to training that only considers the formal study of target language grammar. Moreover, the results also show that thoughts from respondents are formulated on the basis of a single manner of understanding languages that also coincides with what has been experimented in person at the pre-university stages: formal instruction. Nonetheless, from the action research, it appears that respondents satisfactorily embrace the opportunities provided through 'acquisition learning'.
\end{abstract}

Keywords: grammar, language teaching, primary education.

* Doctor en Filología Inglesa, Universidad de Cádiz (España). Correo electrónico: joseluis.estrada@uca.es

** Doctor en Filología Alemana, Universidad de Cádiz (España). Correo electrónico: paco.zayas@uca.es

Cómo citar este artículo: Estrada Chichón, J.L. y Zayas Martínez, F. (2019). Creencias de futuros docentes sobre la gramática en la enseñanza de idiomas. Enunciación, 24(2), 152-168. DOI: http://doi.org/10.14483/22486798.14838

Artículo recibido: 30 de abril de 2019; aprobado: 03 de octubre de 2019 


\section{Introducción}

Este trabajo se centra en las creencias (Farrell, 1999) de estudiantes del grado en Educación Infantil de una universidad pública en España, Universidad de Cádiz, que asumen el posible reto profesional de enseñar inglés como lengua extranjera en etapas tempranas de educación escolar (3 a 6 años). El estudio aborda el posible conflicto metodológico derivado del choque entre sus experiencias previas como "aprendientes" de inglés y las propuestas "adquisitivistas" (Haidl, 1993, 1995) de las corrientes didácticas actuales. La gramática de la lengua meta representa, de forma general, el elemento más comprometido de este contraste, es decir, la medida en que pudiera ser cuestionable una propuesta metodológica que contemplara la atención consciente a aspectos formales de la lengua (Kelly, 1969; Harley, 1989). A este dilema se suma, en el caso de los profesores que ejercen en Educación Infantil, la incuestionable circunstancia de enseñar sin el apoyo de la lengua escrita y sin la posibilidad de plantear explícitamente fórmulas organizativas de los contenidos lingüísticos que se trabajan en el aula. Estas particulares circunstancias técnicas de los profesionales de Educación Infantil, unidas al mencionado conflicto entre sus variadas experiencias de aprendizaje idiomático y la lenta pero inexorable instauración de modelos didácticos adquisitivistas en los centros educativos en España, aconsejan una revisión previa del marco teórico contextual de este estudio. Ante este escenario, los objetivos del presente trabajo son los siguientes:

i. Recopilar las opiniones de los estudiantes matriculados en la asignatura "Didáctica de la lengua extranjera en Educación Infantil: inglés" durante el curso 2018/2019. Esta asignatura queda encuadrada en el tercer curso del grado en Educación Infantil ofertado por la Facultad de Ciencias de la Educación de la Universidad de Cádiz. Las opiniones de los estudiantes se centran en dos temáticas: por un lado, el concepto de gramática, como elemento inherente a los procesos de enseñanza/aprendizaje de lenguas extranjeras; $y$, por otro, las experiencias de los propios estudiantes como aprendientes de inglés durante su formación preuniversitaria (6-18 años, básicamente).

ii. Averiguar las expectativas de los estudiantes sobre cómo quisieran que transcurriesen las prácticas docentes ante el hipotético caso de tener la oportunidad de comenzar una nueva etapa de aprendizaje de una lengua extranjera. El interés reside en conocer de primera mano las creencias que prevalecen entre los informantes en relación con sus preferencias sobre la repetición de determinadas experiencias ya vividas en el pasado como aprendientes de inglés como lengua extranjera.

iii. Conocer, a partir de la investigación-acción, la opinión de los estudiantes acerca de su formación actual en idiomas como parte del objetivo de la asignatura "Didáctica de la lengua extranjera en Educación Infantil: inglés" ("Conocer y poner en práctica los aspectos básicos de la competencia comunicativa en la lengua extranjera en Educación Infantil"; Programa Docente 2018/19, 2019) fundamentada en los principios de la enseñanza adquisitiva (Haidl, 1993, 1995) de lenguas extranjeras que, a su vez, coincide con la tipología de formación en idiomas esperada para Educación Infantil en relación a la supresión de la presentación explícita de normas y reglas gramaticales frente al uso de la lengua extranjera con fines adquisitivos y no (o no solo) instruccionales.

Resulta interesante que los futuros docentes de lenguas extranjeras en etapas educativas tempranas sean conscientes de sus variadas posibilidades metodológicas. Enseñar de una forma natural resulta idóneo para aprendientes que, por una parte, no pueden atender conscientemente cuestiones formales de la lengua porque no dominan el uso 
escrito y, por la otra, no están todavía contaminados por fórmulas de enseñanza/aprendizaje convencionales basadas en la aplicación sistemática de cuestiones teóricas descontextualizadas.

\section{Marco teórico contextual}

\section{Enseñar lenguas extranjeras hoy}

La enseñanza de idiomas puede haberse convertido en el principal argumento de la innovación educativa en los últimos veinte años, concretamente el inglés como LE (Richards, 2016). Todos los niveles del sistema educativo español se han visto afectados por la progresiva aceptación científica, administrativa y social de que las lenguas se "adquieren" mediante el uso y no desde el aprendizaje consciente de sus características formales (Krashen, 1982; Langacker, 1987; Wong-Fillmore, 1989). La transformación paulatina del panorama didáctico en España arranca a finales del siglo pasado, con las reclamaciones de los metodólogos y demás especialistas en enseñanza de lenguas sobre la inutilidad de seguir enseñando las lenguas extranjeras a partir de esquemas clásicos de aproximación, comprensión y aplicación práctica de contenidos (Cook, 2016). La famosa taxonomía de Bloom et al. (1956) se ponía así en entredicho desde las corrientes didácticas de corte cognitivista (Borg, 2003; Achard, 2004), que proponían enfoques adquisitivistas. La aparición, en 2002, del Marco común europeo de referencia para las lenguas: enseñanza, aprendizaje, evaluación (MCERL) supuso posiblemente el hito académico que terminó despejando las posibles dudas de los escépticos y los rezagados, incluyendo aquí muy especialmente las editoriales dedicadas a la publicación de manuales para la enseñanza de lenguas extranjeras (Figueras, 2007, 2012).

Más allá de la homogeneización terminológica para la referencia a niveles progresivos de dominio de lenguas, el MCERL (Consejo de Europa, 2001) ayudó a naturalizar la discusión sobre usuarios (básicos, independientes o competentes), capacidades de comprensión (auditiva o lectora), interacción oral, expresión (oral o escrita), etc., muy en consonancia con la modernización pedagógica general de aquel momento en torno al concepto de competencia (Trim, 2001; Alderson et al., 2004). El eco casi inmediato y sostenido desde entonces en el panorama educativo español fue la proliferación de propuestas educativas de bilingüismo y plurilingüismo (Dobson, Pérez y Johnstone, 2011; Huguet, 2014; Pavón, 2018). Los centros de educación primaria y educación secundaria obligatoria (ESO), en primera instancia, seguidos en la actualidad por las ofertas universitarias de itinerarios académicos bilingües y másteres internacionales impartidos en inglés o EMI (English Medium Instruction) (Doiz, Lasagabaster y Sierra, 2012; Macaro, 2017), así como también por centros de Educación Infantil con propuestas didácticas de lengua extranjera, han transformado radicalmente la intención institucional del aprendizaje de idiomas. Los responsables políticos e institucionales de unos y otros niveles, sus docentes en último término, se afanan en capacitar a los estudiantes para el uso instrumental de lenguas extranjeras. Una cuestión bien distinta es si esta intención institucional es o no por sí misma suficiente para lograrlo: las condiciones pedagógicas (ratio de alumnos por profesor, materiales y recursos didácticos, etc.) y laborales (sistemas de reciclaje profesional, reconocimientos de productividad, etc.), unidas muy especialmente a la capacitación metodológica de los docentes, vienen poniéndose constantemente en entredicho por los usuarios (incluidos aquí los padres de alumnos en etapas preuniversitarias), los metodólogos de enseñanza de lenguas extranjeras y los observadores (la crítica social, encabezada por los medios, que denuncian la falsedad de las propuestas de bilingüismo educativo) (Martín-Arroyo, 2017).

En este contexto socioeducativo irrumpe con fuerza también en los últimos años la revolución pedagógica que conlleva la incorporación de las tecnologías de la información y las comunicaciones (TIC) a las aulas (Chun et al., 2016). En el ámbito de 
la enseñanza de lenguas, aunque no exclusivamente, este proceso deriva en propuestas de blended learning que, por una parte, ofrecen una posibilidad de diversificación de los modelos lingüísticos a que se expone el aprendiente, pero que también exigen una nueva forma de planificación didáctica. Los docentes deben ser ahora conscientes de las posibilidades que ofrece el espacio de aprendizaje presencial para el ensayo (desde el más básico imitativo hasta el más creativo) de la interacción directa (Bocanegra y Haidl, 1999), al tiempo que reservan el espacio de aprendizaje virtual para la exposición a procesos igualmente explorativos de interpretación (primero extensiva y paulatinamente más y más intensiva) o de producción (oral o escrita). En otras palabras, los docentes ya no planifican solamente lo que acontece en el aula, sino que coordinan esto con lo que actualmente puede hacerse fuera de ella, con independencia de si son actividades de recepción o de producción, individuales o cooperativas, etc. Esta nueva circunstancia obliga a los profesores de lenguas extranjeras a reconsiderar el lugar y el modo que ocupa en el proceso de enseñanza/aprendizaje la atención consciente (Schmidt, 1990, 2001; Loewen, 2015; Long, 2017) a los aspectos formales de la lengua meta. Pero, una vez más, ¿están preparados los profesores de/en lenguas extranjeras para enseñar con arreglo a estos nuevos requerimientos?

La llamada enseñanza adquisitiva (Haidl, 1993, 1995) de lenguas extranjeras resume las diversas propuestas didácticas de los enfoques metodológicos de corte cognitivista que, con mayor o menor acierto, abogan por la reproducción en el aula de las condiciones de autenticidad que rodean los procesos de adquisición natural de lenguas (Krashen, 1992). Las propuestas didácticas de adquisición en el aula de los metodólogos entre los años 1990 y la primera década de nuestro siglo (Haidl, 1993, 1995; Zayas, 2002) encuentran actualmente un correlato pedagógico importante en las variadas formas de enseñanza bilingüe y plurilingüe de nuestro entorno (Estrada, 2017). Con independencia del nivel de los participantes, las materias a que se apliquen, o el volumen de presencia de la segunda lengua en el currículo (Julius y Madrid, 2017), etc., las propuestas de enseñanza bilingüe siempre parten del valor vehicular de la lengua meta en el aula (Dalton-Puffer, Nikula y Smit, 2010); esto es, asumen el principio adquisitivista de la autenticidad en el uso (Marsh, 2009) más allá de la mera contextualización simulada de los elementos lingüísticos. Pero ¿quién enseña entonces la lengua?, ¿en qué consiste finalmente enseñar una lengua extranjera?, ¿quién y cómo determina qué tratar primero y qué después?, ¿qué lugar ocupa el aprendizaje de las palabras, de su comportamiento en las estructuras?, ¿qué hacemos con los aspectos formales de la lengua?

\section{El papel de la gramática}

Todas las preguntas con las que atrás cerramos reciben hoy una respuesta demoledora: los profesores no parecen estar preparados (Unesco, 2018) para diseñar sus clases de idiomas con arreglo a los nuevos requerimientos del blended learning para una enseñanza de tipo adquisitivo; de igual modo, ya no son los profesores de lengua los que protagonizan el proceso, sino que son los que enseñan en la lengua extranjera (Coyle, Hood y Marsh, 2010) quienes facilitan su adquisición; y, entre tanto, los profesores de lenguas parecen refugiarse en aquello que los demás no pueden o saben hacer, es decir, utilizar la gramática como eje de sus cursos (Hall, 2017).

El concepto de gramática ha sido una cuestión de debate en relación con su papel en la enseñanza de lenguas extranjeras (Ellis, 2001) que, por otro lado, ha estado siempre ligada al estudio de las reglas y las normas gramaticales de la lengua meta, a pesar de las dudas de los investigadores sobre la consistencia empírica de este hecho (Larsen-Freeman, 2015); en otras palabras, "la enseñanza tradicional todavía parece prevalecer [...] a pesar de los esfuerzos por alejarse de ella" (Jean y Simard, 2011, p. 479; traducción propia). Tanto profesores como estudiantes reconocen la necesidad de enseñar y 
aprender los aspectos gramaticales de una lengua (Griffiths, 2008). Asimismo, el aprendizaje de lenguas extranjeras sigue centrado en ejercicios puramente mecánicos, donde predomina el trabajo con la competencia escrita sobre la oral (Estrada, 2017).

Con independencia del lugar que debe ocupar la gramática (Ellis, 2006) en la enseñanza de lenguas, existe una corriente a favor de la naturaleza cognitiva y no solo instruccional (Calvi, 1999) del proceso didáctico. La enseñanza adquisitiva de lenguas extranjeras, como se expuso en la sección anterior, está íntimamente ligada con la concepción mentalista del lenguaje (Chomsky, 1959). En términos de formación idiomática, esto supone la defensa de los procesos de adquisición frente a los de aprendizaje lingüístico (Corder, 1967; Krashen, 1982). El desarrollo de la gramática de una lengua extranjera es factible, por tanto, si parte de vías de exploración mentales similares a las ocurridas para una lengua materna (Weber, Luther, Indefrey y Hagoort, 2016). La reactivación en el aula de mecanismos cognitivos similares a los activados inicialmente para los procesos de adquisición de lenguas primeras (Larsen-Freeman, 2018) supone que la gramática de una LE cualquiera puede adquirirse por medios distintos a ejercicios mecánicos de repetición.

La concepción tradicionalista de la enseñanza/ aprendizaje de lenguas extranjeras a partir de la presentación explícita de los aspectos gramaticales y su estudio, además de mala prensa (Cook, 1994), conduce a unos resultados deficitarios (EECL, 2012). La aplicación sistemática de actuaciones docentes sin un valor comunicativo real conlleva, en gran medida, que las editoriales establezcan unos objetivos y procedimientos perdurables para un público muy numeroso, y refuerza el conservadurismo de los profesores (Skehan, 1998; Thornbury, 1998). Desde una perspectiva de la secuenciación de la gramática de la lengua meta, esto implica que tanto los libros como los profesores gestionan, por lo general, modelos lineales preestablecidos para el aprendizaje de la lengua (Nunan, 1998). Sin embargo, parece que esta afirmación carece de una fundamentación científica sólida a la luz de la investigación reciente (SaviIle-Troike y Barto, 2017), por lo que no termina de calar en las conciencias profesionales de los docentes. En las secciones siguientes analizamos más de cerca esta cuestión.

\section{Metodología}

En esta sección se presentan los métodos utilizados en relación con los sujetos, los instrumentos y el procedimiento de investigación. En líneas generales, este trabajo representa un estudio de caso perteneciente a la Universidad de Cádiz, situada en la Comunidad Autónoma de Andalucía (España), respecto de las opiniones de estudiantes del grado en Educación Infantil (2018/19), acerca del concepto de gramática como elemento del proceso de enseñanza/aprendizaje de lenguas extranjeras. Asimismo, es un trabajo de investigación cuyo enfoque mixto supone la obtención de datos tanto cuantitativos como cualitativos, por medio de un cuestionario (Cook y Singleton, 2014) y un grupo focal, respectivamente, junto al análisis posterior, y su vinculación con la investigación-acción resultado de la formación idiomática actual de los propios informantes a partir de su participación en la asignatura "Didáctica de la lengua extranjera en Educación Infantil: inglés".

\section{Sujetos}

Los informantes escogidos para este trabajo conforman el grueso del conjunto de estudiantes del grado en Educación Infantil matriculados en la asignatura "Didáctica de la lengua extranjera en Educación Infantil: inglés", en una universidad pública situada en el sur de España: la Universidad de Cádiz. Por tanto, los criterios de selección de la población analizada se restringen a este mismo grupo de estudiantes, de lo que resulta un muestreo de tipo censal, es decir, la muestra es toda la población disponible. El reducido volumen de informantes $(\mathrm{N}=10)$ solo podría haberse aumentado mediante la incorporación de estudiantes de otros 
títulos (por ejemplo, Grado en Educación Primaria), con lo que los resultados sí tendrían un verdadero sesgo cualitativo.

El número de estudiantes matriculados es 12, de los cuales 10 asisten regularmente a clase (porcentaje de asistencia superior al $80 \%$ ), entre los que 9 son mujeres y solamente 1 es hombre. Su rango de edad oscilaba entre 21-25 años, y todos ellos nacieron en Andalucía, comunidad autónoma situada en el sur España en donde se encuentra la provincia de Cádiz, y donde también cursaron todas las etapas de educación media (6-18 años). Solo dos estudiantes son de provincias distintas a Cádiz (Sevilla y Jaén), si bien todos son de la Universidad de Cádiz. La excepción es una alumna que actualmente disfruta de una beca del programa de movilidad Sistema de Intercambio entre Centros Universitarios Españoles (SICUE), cuya procedencia es la Universidad de Jaén, también en Andalucía.

Todos los informantes presentan una formación académica similar, siendo cinco los estudiantes que poseen una acreditación idiomática oficial en lengua inglesa como usuario independiente, según el MCERL: B1 $(\mathrm{N}=4)$ y $\mathrm{B} 2(\mathrm{~N}=1)$. Por último, la asignatura "Didáctica de la lengua extranjera en Educación Infantil: inglés" es la primera del grado en Educación Infantil para todos los informantes en donde el contenido se imparte íntegramente en inglés como lengua extranjera, al menos en lo que compete a la sección Competencia lingüística (práctica) de la asignatura.

\section{Instrumentos}

Los instrumentos para la recopilación de datos que dan forma a este trabajo de investigación son dos: por un lado, se utilizó un cuestionario traducido al español y adaptado del formulario original de Cook y Singleton (2014) sobre el concepto de gramática. El escrito original se estructura en seis secciones, de las cuales cuatro se emplearán para el propósito de este estudio (anexo 1). La primera sección recoge una serie de afirmaciones sobre el concepto de gramática como parte inherente a los procesos de enseñanza/aprendizaje de lenguas extranjeras. Las opciones de los informantes para responder son únicamente tres: "sí", "no" y "no lo sé". Las cuestiones incluidas en la siguiente sección sirven para recopilar información acerca de las experiencias de los propios informantes durante educación media en materia de formación idiomática, así como el grado de frecuencia de exposición a dichas experiencias. El primer bloque de preguntas requiere una respuesta "sí" o "no", mientras que para la segunda sección se utilizó un formato de escala Likert, cuyo rango oscilaba entre 1 (nunca) y 5 (siempre). La tercera sección se confecciona a partir de un conjunto de afirmaciones sobre las circunstancias ideales que darían forma a una instrucción idiomática nueva para estos mismos informantes. La estructura de las preguntas está pensada para recibir información a través de una tipología Likert de respuestas, repitiendo la misma relación de valores que en el apartado anterior: 1 (nunca) y 5 (siempre). La última sección permite escoger una definición del concepto de gramática entre tres opciones (¿conjuntos de patrones para ordenar las palabras en oraciones?; ¿procesos mentales para estructurar el lenguaje?; y ¿conjuntos de patrones para ordenar las palabras en oraciones?) y determinar su importancia como un elemento propio de los procesos de enseñanza/ aprendizaje de idiomas mediante una tipología de pregunta abierta.

En segundo lugar, se desarrolló un análisis de grupo focal dividido en dos sesiones distintas en el que participaron un total de diez estudiantes. El análisis se realizó tras haber valorado las respuestas de los estudiantes en el cuestionario sobre el concepto de gramática. Este instrumento de investigación se categoriza como técnica de investigación de tipología cualitativa, y su objetivo principal es arrojar luces sobre determinadas cuestiones que son difícilmente descifrables por otros medios. Se buscaron respuestas a las dos siguientes preguntas, a saber:

i. Según los resultados del cuestionario, vuestra formación en inglés en educación media se 
fundamentó en un estudio consciente de la gramática de la lengua meta. Además, vuestra concepción ideal sobre cómo os gustaría aprender un nuevo idioma si tuvieseis la oportunidad en la actualidad implica, otra vez, el acto de "recibir explicaciones sobre reglas gramaticales" de una forma explícita. ¿A qué se debe esto?

ii. "En la etapa formativa de Educación Infantil, la metodología para la enseñanza de idiomas no admite la presentación explícita de reglas y normas gramaticales, ni tampoco la lengua escrita, sino el uso de la lengua meta con propósitos auténticos y siempre con fines concretos. ¿Cómo acometéis esta circunstancia, atendiendo a vuestra posición generalizada a favor de 'recibir explicaciones sobre reglas gramaticales'?

La enseñanza adquisitiva de lenguas extranjeras se desmarca de la presentación explícita de reglas y normas gramaticales característica de la instrucción formal. Al contrario, la gramática de una lengua natural representa un constructo mental al que el aprendiente va accediendo paulatinamente por medio de la exposición, primero, y la experimentación, después, con la lengua meta (Corder, 1981). Esto implica que el aprendiente construye sus propias hipótesis acerca del funcionamiento de la estructura interna de la lengua. En cuanto a las sesiones prácticas de la asignatura "Didáctica de la lengua extranjera en Educación Infantil: inglés", se establecieron las dinámicas que se presentan a continuación conforme a los principios metodológicos de la enseñanza adquisitiva. Estos últimos asisten a la investigación/acción que caracteriza al presente trabajo mediante la comprensión de los propios aprendientes de los beneficios lingüísticos que conlleva la enseñanza adquisitiva, junto a su futura aplicación a contextos de Educación Infantil:

1. Preferencia a favor de los procesos subconscientes de adquisición lingüística frente a los procesos de aprendizaje consciente (Krashen, 1982).
2. Ausencia de cualquier tipo de presentación explícita de las normas y las reglas gramaticales del inglés.

3. Empleo exclusivo de la comunicación oral en el aula, otorgando a la lengua escrita un espacio restringido al trabajo personal de cada alumno.

4. Empleo del inglés como única lengua en el aula, otorgando al español un papel limitado a momentos de auténtica necesidad comunicativa.

5. Preferencia de la ausencia de intervención correctiva (Estrada, 2017) frente a técnicas de corrección de errores intervencionistas.

\section{Procedimiento}

El diseño del cuestionario se confeccionó por medio de la aplicación Google Forms. El objetivo apuntaba a facilitar un enlace web a los estudiantes desde el cual acceder al cuestionario a través de sus dispositivos móviles. La última pregunta del cuestionario era la única que requería una explicación más distendida por parte de los informantes, de forma que este medio favorecía la gestión del cuestionario. Todas las respuestas del cuestionario se recopilaron en un plazo de dos días (12 y 13 de febrero de 2019) con el objetivo de evitar el comienzo de las clases presenciales y, con ello, eliminar sesgos en las respuestas.

La distribución y recopilación de datos del cuestionario se llevó a cabo antes del inicio de la asignatura "Didáctica de la lengua extranjera en Educación Infantil: inglés" para evitar cualquier tipo de contaminación: esta asignatura tiene en cuenta los principios teóricos de la enseñanza adquisitiva de lenguas extranjeras, cuyo enfoque práctico aboga por su uso natural, evitando la presentación explícita de conceptos gramaticales y activando los procesos mentales similares a los de la adquisición temprana de una primera lengua.

Previamente al grupo focal, se analizaron todos los datos recopilados en los cuestionarios, presentándole a los participantes los resultados más 
significativos. El grupo focal se desarrolló en dos sesiones el 26 de febrero de 2019, pero de forma contigua, a la que acudieron un total de 10 estudiantes: 3 y 7 , respectivamente. La estructura de la reunión con ellos estaba determinada de antemano, y fue gestionada, en todo momento, por dos moderadores (los autores del presente trabajo). Sin embargo, el desarrollo del grupo focal supuso la puesta en escena de líneas de debate alternativas derivadas de los dos grandes bloques temáticos. Las conversaciones fueron grabadas y transcritas posteriormente para facilitar un análisis objetivo y detallado de las respuestas de los informantes.

El grupo focal tuvo una duración aproximada de 40 minutos (20 minutos para cada sesión), y se llevó a cabo dentro del horario de clases de los estudiantes para facilitar su asistencia. Asimismo, todos ellos se encontraban en un periodo de prácticas en centros escolares de Educación Infantil de la provincia de Cádiz, el cual tenía lugar entre miércoles y viernes, en horario de 9:00-14:00 horas. El lugar escogido para reunirse con los informantes fue el despacho de uno de los investigadores por disponer de un amplio lugar de trabajo. Se utilizó una mesa circular para una cómoda disposición de todos los participantes.

Entre los objetivos del grupo focal, buscábamos recopilar ejemplos concretos de la formación de los informantes en educación media. Pretendíamos averiguar si todos sus profesores habían actuado de la misma forma (o no) en cuanto a la presentación de la gramática en la enseñanza de lenguas extranjeras. Esperábamos que los estudiantes expusieran las razones por las que seguían apostando por una enseñanza de tipo gramatical. Esto permitiría arrojar luz sobre el conflicto de partida de este trabajo de investigación: los estudiantes creen que la gramática no debe ser el eje de los procesos de enseñanza de idiomas; sin embargo, creen que el dominio que ellos mismos tienen de la lengua extranjera se debe al conocimiento consciente que poseen de su gramática. El tono del grupo focal debía facilitar una libre expresión de pensamientos sin entrar en juicios de valor sobre las prácticas docentes recibidas por los estudiantes durante educación media. Se evitaban así sesgos por la manifestación de posturas a favor o en contra de determinadas cuestiones relacionadas con la pedagogía de lenguas.

En último lugar, la asignatura "Didáctica de la lengua extranjera en Educación Infantil: inglés" se encuadra en tercer curso del grado en Educación Infantil. En este curso, los estudiantes acuden únicamente dos días (lunes y martes) a la universidad de manera presencial, mientras que el resto de la semana realizan prácticas en Educación Infantil en colegios de carácter público o privado en la provincia de Cádiz. Esto supone que la extensión de las clases prácticas se limite a 1,5 horas/semana, ajustando a ese tiempo los contenidos obligatorios de la asignatura en lo que compete al "Bloque I: fomento de la competencia lingüística del futuro profesor de inglés en Educación Infantil" (anexo 1). La tabla 1 presenta los campos temáticos trabajados durante las clases prácticas impartidas hasta la fecha de la realización del grupo focal.

Tabla 1. Contenidos de las clases prácticas

\begin{tabular}{l}
\hline \multicolumn{1}{c}{ Sesión 1. Martes, 19 de febrero } \\
\hline [Competencia lingüística (i)] \\
\hline Classroom language \\
\hline Essential grammar in use \\
\hline Campo temático: Classroom \\
\hline Feeling; weather; Numbers; colours \\
\hline \multicolumn{1}{c}{ Sesión 2. Martes, 26 de febrero } \\
\hline [Competencia lingüística (ii)] \\
\hline Classroom language \\
\hline Essential grammar in use \\
\hline Campo temático: Classroom; School \\
\hline Feelings; weather; numbers; colours
\end{tabular}

Fuente: elaboración propia.

Para todas las sesiones prácticas $(\mathrm{N}=9)$ durante el curso 2018/2019, la metodología desarrollada contempla los principios de la enseñanza adquisitiva enumerados en el apartado anterior. Ambas sesiones prácticas, 1 y 2, por su parte, sirven para 
recopilar las opiniones de los aprendientes que, asimismo, a partir de la investigación-acción, suministró a los propios informantes el conocimiento esperado sobre los principios adquisitivistas de la enseñanza de idiomas para tres aspectos principales, a saber: la ausencia de presentación explícita de normas y reglas gramaticales; el empleo de la comunicación oral frente a la escrita; y, por último, el uso exclusivo del inglés (lengua extranjera) frente al español (lengua materna). El resto de sesiones prácticas $(\mathrm{N}=7)$ se desarrolló hasta el mes de mayo de 2019, el cual estaba destinado a trabajar las habilidades docentes de los propios aprendientes como profesores de inglés para la etapa de Educación Infantil.

\section{Resultados}

Las respuestas del cuestionario revelan una formación idiomática de los estudiantes durante educación media basada en el estudio de las reglas y las normas gramaticales del inglés como lengua extranjera. En parte, los estudiantes repetirían las experiencias vividas en materia de enseñanza de idiomas durante su formación idiomática preuniversitaria, si tuvieran la oportunidad de aprender una nueva lengua. No obstante, existen indicios de cambio que serán analizados en la siguiente sección, justificando parte de la discusión de los datos obtenidos a partir de las propias opiniones de los informantes.
Un primer aspecto a tener en cuenta es el promedio de años de aprendizaje del inglés como lengua extranjera por parte de los informantes durante su formación en educación media: 12,7 años. Esta cifra se obtiene atendiendo a la media de años de formación idiomática entre el rango de 10-18 años. Asimismo, solo dos estudiantes están acreditados oficialmente en inglés como lengua extranjera con niveles B1 y B2 según el MCERL, respectivamente. La Universidad de Cádiz, por su parte, exige un nivel mínimo de B1 acreditado oficialmente para la obtención del grado en Educación Infantil.

En lo que al concepto de gramática compete, el $90 \%$ de los informantes opina que "la gramática es importante". Además, el mismo porcentaje considera que "la gramática se puede enseñar de forma directa". La totalidad del grupo $(\mathrm{N}=10)$ defiende que "Ios profesores de idiomas deben explicar gramática". A este respecto, una de las razones más significativas para contemplar la gramática como parte esencial de la enseñanza de idiomas es que "los errores gramaticales son muy importantes", al menos para el $70 \%$ de los informantes.

La tabla 2 presenta los porcentajes de respuestas, por un lado, así como los porcentajes de frecuencia en relación con las situaciones planteadas a los estudiantes en el cuestionario. En líneas generales, entre sus experiencias formativas destaca el estudio formal de la gramática a partir de las explicaciones formales de los profesores (90\%), el

Tabla 2. Prácticas habituales de formación idiomática en educación media (\%)

\begin{tabular}{|c|c|c|c|c|c|c|c|}
\hline & & & \multicolumn{5}{|c|}{ Frecuencia: Nunca-Siempre } \\
\hline & Sí & No & 1 & 2 & 3 & 4 & 5 \\
\hline ¿Estudiaste listas de vocabulario? & 90 & 10 & 0 & 20 & 30 & 10 & 40 \\
\hline ¿Escuchabas al profesor explicando reglas y normas gramaticales? & 90 & 10 & 10 & 10 & 30 & 40 & 10 \\
\hline ¿Repetías frases después del profesor o de una reproducción de audio? & 90 & 10 & 0 & 40 & 30 & 30 & 0 \\
\hline $\begin{array}{l}\text { ¿Hacías repeticiones sobre las que practicar una regla o norma gramatical } \\
\text { con otras palabras? }\end{array}$ & 80 & 20 & 20 & 10 & 50 & 10 & 10 \\
\hline ¿Realizabas actividades en grupo, es decir, con otros compañeros? & 90 & 10 & 0 & 30 & 20 & 20 & 30 \\
\hline $\begin{array}{l}\text { ¿Estabas expuesto a discursos o textos escritos auténticos producidos por } \\
\text { hablantes nativos? }\end{array}$ & 40 & 60 & 30 & 10 & 10 & 40 & 10 \\
\hline
\end{tabular}

Fuente: elaboración propia. 
uso de listas de vocabulario (90\%) y, por último, las repeticiones de las explicaciones (80\%). Cabe indicar también que la mayoría de los informantes respondieron de forma afirmativa a la pregunta sobre si los profesores solían planificar habitualmente actividades grupales durante la clase de idiomas. Sin embargo, dichas prácticas versaban sobre materiales no auténticos que, por otro lado, tampoco estaban reproducidos o escritos por hablantes nativos, según los encuestados (60\%).

Sobre las preferencias actuales de los informantes en cuanto a un hipotético episodio de formación en una nueva lengua extranjera, existe un interés generalizado a favor de la exploración receptiva y productiva de la lengua meta a partir de materiales auténticos (90\%). Impera también una postura favorable hacia una tipología de prácticas docentes en donde se conceda un lugar preferente a la comunicación (60 \%). En esta misma línea, los informantes optan por alternativas diferentes a la memorización de vocabulario (60\%), así como la repetición de palabras o frases $(60 \%)$ en la lengua meta. No obstante, la mayoría (60\%) optaría por continuar recibiendo explicaciones formales sobre las reglas gramaticales de la nueva lengua. En la tabla 3 se desglosan estos datos en forma de promedios de frecuencia entre "nunca" y "siempre".

Por último, la mayoría de estudiantes (50\%) se decanta por definir la gramática como "conjuntos de patrones para ordenar las palabras en oraciones" por delante del resto de opciones facilitadas: "procesos mentales para estructurar el lenguaje" (40\%) y "reglas o normas de comportamiento [lingüístico] que no deberían romperse" (10\%). Sin embargo, todos los informantes defienden el valor de la gramática dentro de los procesos de enseñanza/aprendizaje de extranjeras.

\section{Conclusiones}

Las respuestas del cuestionario, junto a la información resultante del grupo focal, revelan una postura de los informantes a favor de aprender la gramática de la lengua inglesa (u otra lengua extranjera cualquiera) tanto por obligación curricular como por su propia elección. Sin embargo, sus posturas se distancian para otros elementos al respecto de su formación en educación media, por un lado, y las expectativas que mantienen ante una nueva formación idiomática, por otro. Los estudiantes mantienen una actitud positiva ante la posibilidad de recibir una instrucción formal que incluya la presentación explícita de normas y reglas gramaticales de la lengua meta: tal y como revela el cuestionario, esto les permitiría obtener una mayor capacidad para comunicarse adecuadamente.

A pesar de la aparente capacidad de comunicación que otorga a los aprendientes el control de

Tabla 3. Preferencias ante una nueva formación idiomática (\%)

\begin{tabular}{llllll}
\hline & \multicolumn{5}{c}{ Frecuencia: Nunca-Siempre } \\
\hline & $\mathbf{1}$ & $\mathbf{2}$ & $\mathbf{3}$ & $\mathbf{4}$ & $\mathbf{5}$ \\
\hline Comunicándome en grupos en el aula & 0 & 30 & 10 & 40 & 20 \\
\hline Trabajando con textos literarios & 10 & 30 & 20 & 40 & 0 \\
\hline Aprendiendo las palabras de memoria & 10 & 50 & 30 & 10 & 0 \\
\hline $\begin{array}{l}\text { Recibiendo explicaciones sobre reglas } \\
\text { gramaticales }\end{array}$ & 0 & 40 & 10 & 40 & 10 \\
\hline Repitiendo palabras o frases & 10 & 40 & 10 & 30 & 10 \\
\hline $\begin{array}{l}\text { Escuchando y leyendo en una lengua } \\
\text { nativa auténtica }\end{array}$ & 0 & 0 & 10 & 50 & 40 \\
\hline Practicando la repetición de estructuras & 0 & 30 & 50 & 20 & 0 \\
\hline
\end{tabular}

Fuente: elaboración propia. 
las normas y las reglas gramaticales de un idioma, las prácticas docentes en Educación Infantil conllevan obligatoriamente una exploración de la lengua meta que debe mantenerse ajena a la presentación explícita de aspectos gramaticales de la lengua extranjera. En este sentido, los informantes analizados en esta investigación asisten a clases prácticas dentro de la asignatura "Didáctica de la lengua extranjera en Educación Infantil: inglés", cuyas dinámicas de aula evitan la presentación formal de elementos gramaticales del inglés según establece la enseñanza adquisitiva de lenguas extranjeras. Como resultado, los estudiantes acceden por primera vez de forma consciente a un modelo alternativo de trabajo en el aula de idiomas: la adquisición.

\section{Experiencia como aprendientes: la gramática como obligación}

Según los resultados, ha quedado resuelto que la formación idiomática de los informantes en educación media atendía a una tipología de clase magistral (estudiante 3, comunicación personal, 26 de febrero de 2019) fundamentada en el aprendizaje de reglas y normas gramaticales de la lengua inglesa, así como en el estudio de listas de vocabulario, entre otros. Además, durante el grupo focal, fue posible averiguar que la instrucción formal se realizaba por vías explícitas, trabajando la competencia escrita. Al mismo tiempo, las explicaciones se ofrecían, para todos los casos analizados $(N=10)$, en español como lengua materna.

En primer lugar, las rutinas de clase durante las asignaturas de inglés en educación media contemplaban pocas actividades distintas a "ejercicios escritos: el workbook" (estudiante 1, comunicación personal, 26 de febrero de 2019). Dicho de otro modo, "el libro y la pizarra: [el profesor] cogía el libro y lo apuntaba en la pizarra; el profesor seguía siempre el orden del libro" (estudiante 2, comunicación personal, 26 de febrero de 2019). Esto justifica, en parte, que el interés docente giraba en torno a la competencia escrita, aunque sus explicaciones solían producirse mayormente en español: "las explicaciones me las daban en español [(lengua materna)] y luego, a lo mejor, en inglés [(lengua extranjera)] me decían: pues ponte a hacer tal actividad [...]. Pero la explicación de cómo se formaba, en español" (estudiante 3, comunicación personal, 26 de febrero de 2019).

En cuanto a las listas de vocabulario, las palabras se presentaban, según los informantes, de una manera descontextualizada o, en el mejor de los casos, agrupadas por campos temáticos. En todo caso, "las listas [de vocabulario] venían detrás del libro [distribuidas por unidades] con su correspondiente 'rayita' para escribir la traducción" (estudiante 4, comunicación personal, 26 de febrero de 2019). Igualmente, las listas de palabras solían acompañarse de una traducción al español, ya que las pruebas de conocimiento o test incluían preguntas sobre traducciones inglés-español o viceversa: "[en los exámenes no se podía utilizar el español.] Mis exámenes consistían en ejercicios 'sacados' del workbook [...], pero las actividades eran: frase... rellena un 'huequecito'; frase... rellena un 'huequecito', o un texto y cuatro preguntas; o verdadero o falso y el porqué, pero eso sí tenía que fundamentarlo en inglés" (estudiante 3, comunicación personal, 26 de febrero de 2019).

\section{Expectativas ante un nuevo aprendizaje: la gramática como elección}

Los resultados justifican que los propios informantes se desmarcan de algunas de las prácticas experimentadas en etapas preuniversitarias: aprendizaje descontextualizado de palabras, repetición de estructuras lingüísticas con fines memorísticos, etc. No obstante, continúan defendiendo una formación en lengua extranjera basada en las explicaciones formales de las normas y las reglas gramaticales con el objetivo de conseguir una "base para entender" (estudiante 4, comunicación personal, 26 de febrero de 2019) la lengua meta; dicho de otro modo, para los informantes, "hace falta un conocimiento básico para entender la gramática, los saludos..." (estudiante 5, comunicación 
personal, 26 de febrero de 2019). Este contexto replica otras investigaciones realizadas en contextos europeos diferentes al español; por ejemplo: Saaristo (2015) analiza el concepto de gramática a partir de la opinión de 189 estudiantes de la Universidad de Jyväskylä, Finlandia.

En cuanto a la principal razón para recibir explicaciones formales en el aprendizaje de una nueva lengua extranjera, los estudiantes coinciden: "no conocemos otra forma de dar clase. Quizás no es la más efectiva ni la más idónea, pero sí la más rápida" (estudiante 1, comunicación personal, 26 de febrero de 2019). La justificación de este comentario corre a cargo de la informante que se erige como portavoz del grupo: "considero que [...] tener que estar empezando un idioma desde la comprensión constante, de estar hablando diariamente, es algo que requiere mucho más tiempo [...] que: 'así se hace el past perfect' [...] y 'repítelo, memorízalo y mañana te lo sabes'" (estudiante 1, comunicación personal, 26 de febrero de 2019). Los informantes aluden, por tanto, a una labor constante de aprendizaje consciente de la lengua meta. Ante esto, se pregunta a ese mismo informante lo siguiente: "y, ¿te lo sabes?" (haciendo alusión a los tiempos verbales en inglés). Su respuesta resultó tajante: "no, todavía no me sé los verbos irregulares" (estudiante 1, comunicación personal, 26 de febrero de 2019).

\section{Enseñar una lengua en Educación Infantil: sin opción a la gramática}

La aparente obligación de los docentes de enseñar gramática, junto a la necesidad de los propios estudiantes de aprenderla, parece no tener una fundamentación científica sólida. Así, Krashen (2013, p. 109, traducción propia) sostiene que "enseñar -s [(conjugación de la tercera persona del singular de los verbos en inglés)] a los aprendientes iniciales es inútil, ya que se adquiere de forma tardía, y simplificar la información para su exclusión resulta inútil". Este ejemplo serviría para contradecir los aparentes beneficios de la instrucción formal, ante lo que habría que hacer prevalecer la adquisición frente al aprendizaje lingüístico: "los resultados mejoran [solo] ligeramente en términos de competencia idiomática cuando se aprenden conscientemente" (Krashen, 1999, p. 245; traducción propia). Los informantes de este estudio, como resultado de su formación idiomática actual, la cual evita conscientemente alusiones directas a cuestiones formales del inglés como lengua extranjera en forma de normas y reglas gramaticales, indican que "un problema [de la Educación Infantil] es que la segunda lengua [(lengua extranjera)] se presenta como algo específico en English hours" (estudiante 1, comunicación personal, 26 de febrero de 2019).

Este posicionamiento supone el rechazo de la enseñanza de idiomas como estudio de la gramática a partir de un proceso de memorización de las características formales de la lengua extranjera; en otras palabras, la primera acepción de enseñanza de idiomas atiende a la supuesta necesidad de los informantes por aprender las reglas gramaticales del inglés para poder comunicarse adecuadamente. Sin embargo, la posibilidad de comunicarse en una lengua extranjera cualquiera no depende exclusivamente de una competencia gramatical concreta (Nikula, 2003). En todo caso, el individuo debe ser consciente de su situación presente como hablante interlengual (Selinker, 1972), o bien le compete al profesor conocer el estado de la interlengua del propio aprendiente. Esto implica que ningún individuo dispondrá de un dominio completo de las reglas y las normas gramaticales de la lengua meta (Krashen, 1982), por lo que, incluso, el estudio de la gramática debería ser suprimida de la enseñanza de idiomas (Krashen, 1993a, 1993b). Uno de los informantes señaló, a este respecto: "si reflexiono ahora, veo que los niños aprenden [un idioma] de otra forma y, si volviera a hacer el cuestionario, rectificaría" (estudiante 2, comunicación personal, 26 de febrero de 2019).

En resumen, los informantes consideran que su formación actual basada en los principios de la enseñanza adquisitiva de lenguas extranjeras les está proveyendo de las estructuras y las normas 
gramaticales del inglés de una manera implícita: "indirectamente estamos dando gramática" (estudiante 6, comunicación personal, 26 de febrero de 2019). Asimismo, contemplan una alternativa metodológica de enseñanza de idiomas apropiada para la etapa de Educación Infantil, lo que les ha aportado "otra formar de enseñar [lenguas extranjeras]" (estudiante 2, comunicación personal, 26 de febrero de 2019). Los aprendientes agradecen la "inmersión total y [la apuesta por] la oralidad" (estudiante 3, comunicación personal, 26 de febrero de 2019), aunque también consideran que les "cuesta prescindir de la forma escrita" (estudiante 2, comunicación personal, 26 de febrero de 2019). Sin embargo, la etapa de Educación Infantil no contempla la lengua escrita, por lo que los informantes muestran su satisfacción por "estar cogiendo ideas" (estudiante 4, comunicación personal, 26 de febrero de 2018), tal y como se detalla entre los objetivos de la asignatura "Didáctica de la lengua extranjera en Educación Infantil: inglés".

En relación con lo presentado hasta aquí, el papel de la gramática en la enseñanza de lenguas y, con él, el papel del docente, continúan siendo uno de los temas centrales de discusión metodológica desde la aparición del enfoque comunicativo (communicative approach). En algunos sectores vinculados a la investigación en enseñanza de lenguas pudimos darnos inicialmente por satisfechos cuando, con la aparición de los enfoques adquisitivistas -incluyendo aquí lo que se conoce por educación bilingüe-, parecía posponerse el tratamiento de la gramática hasta haber experimentado suficientemente con la lengua, apelando a una nueva forma de tratarla. Sin embargo, como revelan las aportaciones de nuestros estudiantes en este estudio, la forma "comunicativa" de abordar la gramática implicó nuevas contradicciones de carácter metodológico: la gramática podía no ofrecerse como punto de arranque de las unidades didácticas del manual con el que se trabajara, pero sí seguía apareciendo como punto de cierre, en forma de resumen, o de tabla de síntesis, etc., de cada tema; de igual modo, podía no requerirse una explicitación formal de la gramática, o la presentación de las reglas, pero sí su correcta aplicación. En esta misma línea, la gramática podía no ser ya tampoco el elemento central de cada secuencia didáctica, donde tendría que convivir con cuestiones culturales, de pronunciación, etc. Por último, la gramática podía dejar de ser el foco principal de las correcciones de los profesores, que se centrarían desde entonces en las desviaciones que impidieran la comunicación; etc.

Pese a todo esto, tal y como se constata en este estudio, seguimos encontrando que los cursos de idiomas se diseñan a partir del reconocimiento de fórmulas de avance gramatical conforme a unidades didácticas presentadas de forma secuencial y de manera muy homogénea. La lengua escrita, en este mismo contexto, sigue ocupando el mayor espacio en los procesos formativos reglados y los docentes pueden, llegado el caso, prescindir totalmente de atender la oralidad. Y, para cerrar la revisión de lo que sucede actualmente en las aulas, los informantes desvelan que la evaluación sigue consistiendo en la medición de momentos de desviación de la norma, que se utiliza para penalizar al individuo con una reducción de la potencial calificación final.

Como consecuencia, los estudiantes siguen pensando que solo se llega a dominar una lengua y, con ello, a comunicarse adecuadamente, a través del estudio consciente de la gramática. Nuestros informantes desconfían en principio de procesos de enseñanza/aprendizaje donde la lengua escrita no desempeñe un papel relevante, es decir, en su inminente incorporación al mundo laboral como profesores de Educación Infantil, ofrecerán resistencia al abandono de las mismas fórmulas didácticas que ellos experimentaron como aprendientes de idiomas en etapas preuniversitarias. De esta manera, continuarán renunciando a activar en sus futuros alumnos una intuición lingüística, en términos chomskianos: creen que su trabajo consistirá en introducir de forma lúdica cuestiones básicas como números, colores, nombres de animales, saludos y rutinas muy básicas, etc., en el mejor de los casos en sensibilizar para el posterior aprendizaje de lenguas en etapas superiores. 


\section{Reconocimientos}

El presente artículo es producto de la investigación derivada del proyecto de innovación concedido en la convocatoria de Actuaciones Avaladas para la Mejora de la Docencia 2015-2016 de la Universidad de Cádiz titulado "Blended Learning: E/ALAO como estrategia de autonivelación para la competencia gramatical en estudiantes de Grado de Magisterio y MAES" (ref.: sol-201500054623-tra), coordinado por el profesor Manuel Francisco Romero Oliva y vinculado al Proyecto Lingüístico de Centro (PLC) de la Facultad de Ciencias de la Educación, donde se integran las estrategias de innovación educativa para el tratamiento de las lenguas extranjeras en la enseñanza AICLE en Educación Superior. Los resultados directos del citado proyecto se publicaron en los trabajos de Jiménez Fernández, Romero Oliva y Heredia Ponce (2019) y Romero Oliva, Jiménez Fernández y Heredia Ponce (2019).

\section{Referencias bibliográficas}

Achard, M. (2004). Grammatical Instruction in the Natural Approach: A Cognitive Grammar View. En M. Achard y S. Niemeier (eds.), Cognitive Linguistics, Second Language Acquisition, and Foreign Language Teaching (pp. 165-194). Berlín: Mouton de Gruyter.

Alderson, J.C., Figueras, N., Kuijper, H., Nold, G., Takala, S. y Tardieu, C. (2004). The Development of Specifications for Item Development and Classification within the Common European Framework of Reference for Languages: Learning, Teaching, Assessment. Reading and Listening. Lancaster: Universidad de Lancaster.

Bloom, B., Engelhart, M., Furst, E., Hill, W. y Krathwohk, D. (1956). Taxonomy of Educational Objectives: The Classification of Educational Goals. Nueva York: David McKay Company.

Bocanegra, A.M. y Haidl, A. (1999). Language Learner Autonomy in Practice: Possibilities in a Foreign Language Situation. Revista Alicantina de Estudios Ingleses, 12, 7-17.
Borg, S. (2003). Teacher Cognition in Language Teaching. A Review of Research on what Language Teachers Think, Know, Believe, and so. Language Teaching, 36(2), 81-109.

Calvi, M.V. (1999). La gramática en la enseñanza de las lenguas afines. En M.C. Losada Aldrey, J.F. Márquez Caneda y T.E. Jiménez Juliá (eds.), Actas del IX Congreso Internacional ASELE. Español como LE, Enfoque Comunicativo y Gramática (pp. 353-360). Santiago de Compostela: Servicio de Publicaciones de la Universidad de Santiago de Compostela.

Chomsky, N. (1959). Review of Skinner's Verbal Behavior. Language, 35, 26-58.

Chun, D., Smith, D. y Kern, R. (2016). Technology in Language Use, Language Teaching, and Language Learning. Modern Language Journal, 100, 64-80.

Consejo de Europa (2001). Marco común europeo de referencia para las lenguas: aprendizaje, enseñanza, evaluación. Traducido por el Instituto Cervantes. Madrid: Anaya.

Cook, V. (1994). The Role of Grammar in Language Teaching. Recuperado de http://www.viviancook.uk/ Writings/Papers/GhentGrammar.htm

Cook, V. (2016). Second Language Learning and Teaching. 5a. ed. Londres: Routledge.

Cook, V. y Singleton, D. (2014). Key Topics in Second Language Acquisition. Bristol: Multilingual Matters.

Corder, P. (1967). The Significance of Learners' Errors. International Review of Applied Linguistics in Language Teaching (IRAL), 5, 160-170.

Corder, P. (1981). Error Analysis and Interlanguage. Oxford: Oxford University Press.

Coyle, D., Hood, P. y Marsh, D. (2010). Content and Language Integrated Learning. Cambridge: Cambridge University Press.

Dalton-Puffer, C., Nikula, T. y Smit, U. (eds.) (2010) Language Use and Language Learning in CLIL Classrooms. Ámsterdam: John Benjamins.

Dobson, A., Pérez Murillo, M.D. y Johnstone, R. (2011). Bilingual Education Project (Spain): Evaluation Report Online Supplement. España: British Council, Ministerio de Educación. Recuperado de https://www. britishcouncil.es/sites/default/files/bilingual-education-project-spain-evaluation-report-en.pdf 
Doiz, A., Lasagabaster, D. y Sierra, J.M. (2012). English-Medium Instruction at Universities Global ChaIlenges. Bristol: Multilingual Matters.

Ellis, R. (ed.) (2001). Form-Focused Instruction and Second Language Learning. Oxford: Blackwell.

Ellis, R. (2006). Current Issues in the Teaching of Grammar: An SLA Perspective. TESOL Quarterly, 4(1), 83-107.

Estrada, J.L. (2017). Tratamiento de errores en la enseñanza de lenguas extranjeras en la educación superior: posibilidades de corrección no intervencionista en la producción oral. [Tesis doctoral]. Universidad de Cádiz, España.

Farrell, T. (1999). The Reflective Assignment: Unlocking Pre-Service English Teachers' Beliefs on Grammar Teaching. RELC Journal, 30(2), 1-17.

Figueras, N. (2007). The CEFR, A Lever for the Improvement of Language Professionals in Europe. The Modern Language Journal, 91(4), 673-675.

Figueras, N. (2012). The Impact of the CEFR. ELT Journal, 66(4), 477-485.

Griffiths, C. (2008). Lessons from Good Language Learners. Cambridge, Cambridge University Press.

Haidl, A. (1993). La "teoría de la relevancia" y los procesos de adquisición en la enseñanza de idiomas extranjeros. Pragmalingüística, 1, 367-398. RODIN, Universidad de Cádiz.

Haidl, A. (1995). Aportaciones a la teoría de la adquisición: una metodología del alemán para universitarios españoles. (Tesis doctoral). Universidad de Cádiz, Cádiz.

Hall, G. (2017). Exploring English Language Teaching: Language in Action. Routledge Introductions to Applied Linguistics. Londres: Routledge.

Harley, B. (1989). Functional Grammar in French Immersion: A Classroom Experiment, Applied Linguistics, 10(3), 331-359.

Huguet, A. (2004). Bilingual Education in Spain: Current Situation and Future Perspectives. Cultura y Educación, 16(4), 399-418.

Jean, G. y Simard, Q. (2011). Grammar Teaching and Learning in L2: Necessary, but Boring? Foreign Language Annals, 44(3), 467-494.
Jiménez Fernández, R., Romero Oliva, M. y Heredia Ponce, H. (2019). Formación inicial del maestro y competencia gramatical para su práctica docente. Revista de Humanidades, 37, 151-178.

Julius, S. M. y Madrid, D. (2017). Diversity of Students in Bilingual University Programs: A Case Study. The International Journal of Diversity in Education, 17(2), 17-28.

Kelly, L. (1969). Five centuries of language teaching: an inquiry into the science, art, and development of language teaching methodology. Rowley, Massachusetts: Newbury House Publishers.

Krashen, S. (1982). Principles and Practice in Second Language Acquisition. Oxford: Pergamon.

Krashen, S. (1992). Fundamentals of Language Education. Beverly Hills: Laredo Publishing.

Krashen, S. (1993a). Teaching Issues: Formal Grammar Instruction. Another Educator Comments. TESOL Quarterly, 26(2), 409-441.

Krashen, S. (1993b). The Effect of Formal Grammar Teaching: Still Peripheral. TESOL Quarterly, 26(3), 722-725.

Krashen, S. (1999). Seeking a Role for Grammar. A Review of Some Recent Studies. Foreign Language Annals, 32(2), 245-254.

Krashen, S. (2013). Should We Teach Strategies? Electronic Journal of Foreign Language Teaching, 10(1), 35-39.

Langacker, R.W. (1987). Foundation of Cognitive Grammar. Theoretical Prerequisites. Stanford: Stanford University Press.

Larsen-Freeman, D. (2015). Research into Practice: Grammar Learning and Teaching. Language Teaching, 48(2), 263-280.

Larsen-Freeman, D. (2018): Looking Ahead: Future Directions in, and Future Research into, Second Language Acquisition. Foreign Language Annals, 21(1), 55-72.

Loewen, S. (2015). Introduction to Instructed Second Language Acquisition. Nueva York: Routledge.

Long, M. (2017). Instructed Second Language Acquisition: Geopolitics, Methodological Issues, and some major Research Questions. Instructed Second Language Acquisition, 1, 7-44. 
Macaro, E. (2017). English Medium Instruction: Global Views and Countries in Focus, Language Teaching, 50(3), 1-18.

Marsh, D. (2009) CLIL: An interview with Professor David Marsh. International House Journal of Education and Development, 26. Recuperado de http://ihjournal.com/content-and-language-integrated-learning

Martín-Arroyo, J. (8 de octubre de 2017). Las sombras del bilingüismo. El País. Recuperado de https://elpais.com/politica/2017/10/06/actualidad/1507284770_581444.html

Ministerio de Educación, Cultura y Deporte (2012). Estudio Europeo de Competencia Lingüística (EECL). Volumen I. Informe Español. Madrid: Secretaría General Técnica. Centro de Publicaciones. Recuperado de http://www.mecd.gob.es/ dctm/ievaluacion/internacional/eeclvolumenii. pdf?documentld=0901e72b813ac516

Nikula, T. (2003). English as an Object and Tool of Study: A Look at Classroom Interaction. En M. Koskela y N. Pilke (eds.), Language and Expertise (pp. 135-157). Jyväskylä, Finlandia: AFinLA.

Nunan, D. (1998). Teaching Grammar in Context. ELT Journal, 52(2), 101-109.

Pavón, V. (2018). La controversia de la educación bilingüe en España. Revista Tribuna Norteamericana, 26, 20-27.

Programa Docente 2018-19 (2019). Asignatura "Didáctica de la lengua extranjera en Educación Infantil: inglés". Manuscrito no publicado. Cádiz, España: Facultad de Ciencias de la Educación. Universidad de Cádiz.

Richards, J. (2016). Key Issues in Language Teaching. Cambridge: Cambridge University Press.

Romero Oliva, M., Jiménez Fernández, R. y Heredia Ponce, H. (2019). Análisis de la implementación de un programa educativo basado en la metodología mobile learning. Texto Livre: Linguagem e Tecnologia, 12(2), 172-201.

Saaristo, P. (2015). Grammar is the Heart of Language: Grammar and its Role in Language Learning among Finnish University Students. En J. Jalkanen, E. Jokinen, y P. Taalas (eds.), Voices of Pedagogical Development - Expanding, Enhancing and Exploring
Higher Education Language Learning (pp. 279-318). Dublin: Research-publishing.net.

Saville-Troike, M. y Barto, K. (2017). Introducing Second Language Acquisition (Cambridge Introductions to Language and Linguistics). Cambridge: Cambridge University Press.

Schmidt, R. (1990). The Role of Consciousness in Second Language Learning. Applied Linguistics, 11, $129-58$.

Schmidt, R. (2001). Attention. En P. Robinson (ed.), Cognition and Second Language Instruction (pp. 3-32). Cambridge: Cambridge University Press.

Selinker L (1972). Interlanguage, International Review of Applied Linguistics in Language Teaching (IRAL), 10, 209-231.

Skehan, P. (1998). A Cognitive Approach to Language Learning. Oxford: Oxford University Press.

Thornbury, S. (1998). Grammar, Power and Bottled Water. IATEFL Newsletter, 140, 19-20.

Trim, J. (2001). The Work of the Council of Europe in the Field of Modern Languages, 1957-2001. Graz: European Centre for Modern Languages. Recuperado de http://archive.ecml.at/efsz/files/Trim.pdf

Unesco (Organización de las Naciones Unidas para la Educación, la Ciencia y la Cultura) (2018). World's Teachers' Day: Quality Education Requires Well-Trained Teachers. Recuperado de https://en.unesco. org/news/world-teachers-day-quality-education-requires-well-trained-teachers

Weber, K., Luther, L., Indefrey, P. y Hagoort, P. (2016). Overlap and Differences in Brain Networks Underlying the Processing of Complex Sentence Structures in Second Language Users Compared to Native Speakers. Brain Connectivity, 6(4), 345-355.

Wong-Fillmore, L. (1989). Language Learning in Social Context: The View from Research in Second Language Learning. En R. Dietrich y C. Graumann (eds.), Language Processing in Social Context (pp. 277-302). Ámsterdam: Elsevier.

Zayas Martínez, F. (2002). La literatura en la enseñanza del alemán como segunda lengua extranjera. Aplicación sistemática de la literatura en niveles iniciales de adquisición. [Tesis doctoral]. Universidad de Cádiz, España. 


\section{Anexo 1. Contenidos}

\section{BLOQUE I: \\ FOMENTO DE LA COMPETENCIA LINGÜÍSTICA DEL FUTURO PROFESOR DE INGLÉS EN EDUCACIÓN INFANTIL.}

1.1. El discurso del aula: "classroom language"

1.2. Campos semánticos básicos que se abordarán en Educación Infantil: my school, my body, my family, colours, shapes, jobs, food, clothes, animals, weather, etc.

1.3. Competencia gramatical: "Essential grammar in use"

\section{BLOQUE II: DIDÁCTICA DEL INGLÉS EN EDUCACIÓN INFANTIL.}

2.1. Diseño curricular en lengua extranjera (inglés) en Educación Infantil: la programación.

2.2. Teorías de adquisición y métodos de enseñanza/aprendizaje de la lengua extranjera en los niveles iniciales.

2.3. Tratamiento de las destrezas y funciones de la Lengua en Educación Infantil.

2.4. Rutinas del aula en L2: asamblea, relajación...

2.5. Psicomotricidad y movimiento en la clase de inglés.

2.6. Materiales y recursos didácticos para la enseñanza del inglés en Educación Infantil.

2.7. Aproximación lúdica a la enseñanza del inglés en Educación Infantil: los juegos, cuentos, dramatización... 2.8. Aplicación del folclore infantil anglosajón a la enseñanza/aprendizaje del inglés en Educación Infantil: nursery rhymes, games rhymes, children's songs. 